\title{
Frequency, Complications, and Predictive Factors for Performing Subtotal Laparoscopic Cholecystectomy in a Hepatobiliary Unit: A Comparative Cohort Study
}

Alexia Farrugia ${ }^{1}$, Niranjan Ravichandran ${ }^{2}$, Majid Ali $^{3}$, Harry Blege ${ }^{4}$, Saboor Khan ${ }^{5}$, For Tai Lam ${ }^{6}$, Jawad Ahmad ${ }^{7}$, Gabriele Marangoni ${ }^{8}$

\begin{abstract}
Aims: Laparoscopic subtotal cholecystectomies (LSCs) are occasionally performed for difficult gallbladder (GB) surgery. The aim of this study is to determine the rate, complications, and factors predictive of performing LSC in a hepatobiliary (HPB) unit, in comparison to patients who have undergone a conventional operation.

Materials and methods: A 5-year retrospective review of laparoscopic cholecystectomies (LCs) was performed by HPB team at a tertiary center. Demographic, operative, and postoperative data were identified. A randomized group (generated using online randomization software Research Randomizer ${ }^{\oplus}$ ) of LC patients was identified from the study cohort, who had the same data recorded for comparison. Significance level was set at $p<0.05$ when comparing the two groups of LC and LSC.

Results: A total of 1,613 patients underwent LC, of which, 102 (6.3\%) underwent LSC. The complication rate was $12.7 \%$ in the LSC group, mainly consisting of bile leak (3.9\%) and collection requiring drainage $(0.98 \%)$. The LC group had a $4.9 \%$ complication rate, of which, one bile leak was reported, i.e., $1(0.98 \%)$. The length of stay was significantly longer in the LSC group ( 2 days vs 0 days in the LC group), and this group also had a slightly higher readmission rate (8.8\% vs $3.92 \%)$. Laparoscopic subtotal cholecystectomy was found to be more likely in patients with previous cholecystitis, thickened GB wall on imaging and previous endoscopic retrograde cholangiopancreatography (ERCP).

Conclusion: Laparoscopic subtotal cholecystectomy is a safe procedure and the above characteristics may be used to potentially predict who is more likely to undergo LSC. This may aid in the consenting process and also help to create a score that predicts the probability of undergoing LSC. Keywords: Gallbladder, Laparoscopic cholecystectomy, Predictive factors, Subtotal cholecystectomy.

World Journal of Laparoscopic Surgery (2019): 10.5005/jp-journals-10033-1369
\end{abstract}

\section{INTRODUCTION}

Since the 1980s, LC has been the gold standard procedure to treat benign gallbladder (GB) disease. This allows for a shorter hospital stay and operating time as well as quicker postoperative rehabilitation and lower wound infection rates. ${ }^{1}$ However, in some cases, such as adhesion, fibrosis, Mirizzi's syndrome, biliary anomalies, empyema of the GB, or gangrene, dissection of Calot's triangle may be very difficult. Previously, this used to be dealt with by converting to an open procedure, which prolongs hospital stay and could be associated with higher complication rates. ${ }^{2}$ This led to the development of laparoscopic cholecystectomies (LSC), which may be attempted to avoid injury to the bile ducts and, therefore, allow a difficult cholecystectomy to still be done as a day-case procedure or potentially with only overnight stay. ${ }^{3,4}$

There are several ways to perform LSC. One method is to excise the anterior wall of the GB with stone extraction and leave the posterior wall attached to the liver. Another method is fundus-first excision of the GB followed by division at the Hartmann's pouch. The cystic duct stump can be either left open or closed using endoloop, an intracorporeal stitch, or stapled. The mucosa of the posterior wall of the remnant GB is diathermied or left alone with or without a drain in situ. ${ }^{5}$

The LSC has been shown to reduce the need for conversion to an open procedure, thus reducing the length of stay and other complications associated with the open cholecystectomy. ${ }^{6}$ Studies have also shown that this procedure decreases the bile duct injury rate $^{3,7-9}$ and is safe in cirrhotic patients. ${ }^{10}$ The aim was to determine
${ }^{1}$ Department of HPB Surgery, University Hospitals Coventry and Warwickshire, Coventry, West Midlands, UK

${ }^{2-8}$ Department of Surgery, University Hospitals Coventry and Warwickshire, Coventry, West Midlands, UK

Corresponding Author: Gabriele Marangoni, Department of Surgery, University Hospitals Coventry and Warwickshire, Coventry, West Midlands, UK, Phone: +44 2476965269, e-mail: Gabriele.marangoni@ uhcw.nhs.uk

How to cite this article: Farrugia A, Ravichandran N, Ali M, et al. Frequency, Complications, and Predictive Factors for Performing Subtotal Laparoscopic Cholecystectomy in a Hepatobiliary Unit: A Comparative Cohort Study. World J Lap Surg 2019;12(2):53-55.

Source of support: Nil

Conflict of interest: None

the rate of LSC and its associated complications in a HPB unit and also to determine the predictive factors for performing LSC, if any.

\section{Materials and Methods}

The study was registered with the local authorities (audit number 215). All patients who underwent LC between 2013 and 2017 at a tertiary center were retrospectively reviewed to determine the frequency of LSC. The data for the initial patient cohort was obtained from the hospital coding team, and patients who underwent LSC were extracted from the operation notes. The inclusion criteria were 
patients over the age of 18 , who had a cholecystectomy, however, excision of the GB was performed at the level of Hartmann's pouch or the posterior wall of the GB was left in situ. Patients under the age of 18 or in cases where method that had been used was unclear were excluded from the study. Data were collected from electronic patient records, regarding age, gender, indication for surgery, procedure done (either LC or LSC), preoperative liver function tests, ultrasound scan results, common bile duct (CBD) dilatation, $\mathrm{BMI}, \mathrm{GB}$ thickness on ultrasound, other investigations such as endoscopic retrograde cholangiopancreatography $(\mathrm{ERCP})$, magnetic resonance cholangiopancreatography (MRCP), or hepatobiliary iminodiacetic acid (HIDA) (scintigraphy) scans, intraoperative details, length of stay, and postoperative complications.

A matched, randomized sample was drawn from the LC cohort, using Research Randomizer ${ }^{\oplus}$. The two groups, LSC and LC, were compared to determine that characteristics, if any, that could predict the need for LSC and whether the groups differed with respect to complications and length of stay. Odds ratios were calculated to assess the risk of patients having a subtotal cholecystectomy. These were assessed using IBM SPSS V23.

\section{Results}

A total of 1,613 patients underwent LC in the HPB unit during this period, of which, 102 (6.3\%) had an LSC (55 females and 47 males) and $4(0.24 \%)$ were converted to open surgery. Thirty-six (2.23\%) of these LSCs were done in the acute setting for cholecystitis, while only 16 (15.7\%) of the LC group were done acutely.

Indications for LSC were acute cholecystitis (56 patients, $54.9 \%$ ), gallstones causing biliary colic (25 patients $24.5 \%$ ), gallstone pancreatitis (10 patients, 9.8\%), cholangitis (6 patients, $5.9 \%$ ), Mirizzi's syndrome (2 patients, 1.96\%), CBD stones (2 patients $1.96 \%$ ), and acalculous cholecystitis (1 patient, $0.98 \%$ ). Indications for LC were gallstones causing biliary colic (66 patients $64.7 \%$ ), acute cholecystitis (21 patients, 20.6\%), gallstone pancreatitis (13 patients, $12.7 \%$ ), dyskinesia (1 patient, $0.98 \%$ ), and GB polyp (1 patient, $0.98 \%$ ). Complications in each group are shown in Table 1.

Odds ratios were calculated to assess the correlation between several characteristics and the likelihood of having LSC rather than LC. We can see that previous cholecystitis, thickened GB wall on ultrasound scan, and previous ERCP led to a higher likelihood that subtotal cholecystectomy would be required. These are shown in Table 2.

Table 1: Indication for LSC were acute cholecystitis are shown in each group

\begin{tabular}{lllr}
\hline & LSC & LC & p value \\
\hline Complications & $13(12.7 \%)$ & $5(4.9 \%)$ & $<0.001$ \\
Bile leak & $4(3.9 \%)$ & $1(0.98 \%)$ & $<0.001$ \\
Collection (with drainage) & $1(0.98 \%)$ & 0 & $<0.001$ \\
CBD injury & 0 & $1(0.98 \%)$ & $<0.001$ \\
Retained stone & $1(0.98 \%)$ & 0 & $<0.001$ \\
Gastric ulcer & $2(1.96 \%)$ & 0 & $<0.001$ \\
Hospital-acquired pneumonia & $1(0.98 \%)$ & 0 & $<0.001$ \\
Ileus & $1(0.98 \%)$ & 0 & $<0.001$ \\
Bleeding & 0 & $2(1.96 \%)$ & $<0.001$ \\
Readmission & $9(8.8 \%)$ & $4(3.92 \%)$ & 0.526 \\
Other complications & $3(2.94 \%)$ & $1(0.98 \%)$ & $<0.001$ \\
Median length of stay/days (IQR) & $2(2-3)$ & $0(0-1)$ & $<0.001$ \\
\hline
\end{tabular}

LSC, laparoscopic subtotal cholecystectomy; LC, laparoscopic cholecystectomy; CBD, common bile duct; IQR, interquartile range. $p<0.05$ was considered statistically significant

\section{Discussion}

The main use of a subtotal cholecystectomy is in the context of acute cholecystitis or repeated cholecystitis where inflammation and fibrosis may make safe dissection of Calot's triangle more difficult. In such a situation, conversion to open procedure is more common, and thus subtotal cholecystectomy may be of use in situations where cholecystectomy for acute inflammation is slightly delayed. ${ }^{11}$ However, there have been reports of longer operating times ${ }^{12}$ and more blood loss ${ }^{13,14}$ as well as increased hospital stay ${ }^{14}$ when compared to normal cholecystectomy. Our data confirms a slightly higher complication rate and longer hospital stay. However, other studies have shown no differences in complications and hospital stay, ${ }^{15}$ and a meta-analysis has found that the complication rates for subtotal cholecystectomy are similar to total cholecystectomy, so that it can be made a simple and effective procedure for difficult GB. ${ }^{16}$

When compared with procedures converted to open cholecystectomy, patients with LSC had more bile duct injury, less wound infection, shorter hospital stay, more recurrent biliary events, more postoperative ERCP, and a higher reintervention rate. ${ }^{17}$ It is, however, less complicated than open cholecystectomy and has decreased costs. ${ }^{18}$ Another possible complication of LSC is retention of stone within the Hartmann's pouch, which may even require reoperation, though this was not seen in our cohort. ${ }^{19}$

No defined risk factors were reported for subtotal cholecystectomy, though there have been associations with high C-reactive protein (CRP) and Tokyo grading. ${ }^{20}$ Shingu et al. attempted to create a predictive score for LSC which consisted of preoperative CRP elevation, wall thickened GB, atrophic GB, pericholecystic abscess, and structure of the hepatic bile duct. Mean of the predictive score in LSC was 8.2, and ideal cutoff point for score was 8 ; specificity and sensitivity toward LSC was $76.0 \%$ and $77.0 \%$, respectively. ${ }^{21}$ However, the use of such a score has not been widely implemented. We have seen that our study partially corroborates this, as patients who had previous cholecystitis, thickened GB wall on imaging, and previous $\operatorname{ERCP}(p<0.01)$ were more likely to require subtotal cholecystectomy. This may be related to increased local inflammation in the area causing difficulty in dissecting Calot's triangle in patients with several episodes of cholecystitis and those requiring $E R C P$.

\section{Conclusion}

Laparoscopic subtotal cholecystectomy is a safe procedure to perform in cases of difficult cholecystectomy, despite a slightly higher length of stay and complication rate than LC. It is more likely in patients with repeated episodes of cholecystitis, requiring previous ERCP or having a thickened GB wall. In the future, these characteristics may be used in the formation of a predictive score

Table 2: Predictive factors for subtotal cholecystectomy

\begin{tabular}{ll}
\hline Preoperative characteristic & Odds ratio $(95 \% \mathrm{Cl})$ \\
\hline Jaundice & $1.8(0.9-3.6)$ \\
Pancreatitis & $0.8(0.4-1.9)$ \\
Cholecystitis & $4.3(2.3-8.0)$ \\
Thickened gallbladder wall & $6.1(3.3-11.1)$ \\
ERCP & $4.7(2.2-9.9)$ \\
BMI $>30$ & $1.2(0.7-2.1)$ \\
\hline
\end{tabular}

$\mathrm{Cl}$, confidence interval; $\mathrm{BMI}$, body mass index 
for subtotal cholecystectomy, also allowing the surgeon to obtain patient's consent prior to the operation; however, more work is required for such a score to be finalized.

\section{Clinical Significance}

An LSC may be a way to avoid converting to an open procedure, as the complication rate and length of stay are only slightly higher than a normal LC. Certain factors such as previous inflammation and ERCP may mean that a patient may be more likely to need a laparoscopic subtotal cholecystectomy. With more data, a predictive score may be formed.

\section{References}

1. Vracko J, Hunt MY, Wiechel KL. Safe laparoscopic cholecystectomy. Surg Endosc 2005;19(12):1666. DOI: 10.1007/s00464-004-2138-y.

2. Wolf AS, Nijsse BA, Sokal SM, et al. Surgical outcomes of open cholecystectomy in the laparoscopic era. Am J Surg 2009;197(6): 781-784. DOI: 10.1016/j.amjsurg.2008.05.010.

3. Beldi G, Glattli A. Laparoscopic subtotal cholecystectomy for severe cholecystitis. Surg Endosc 2003;17(9):1437-1439. DOI: 10.1007/s00464002-9128-8.

4. Krahenbuhl L, Sclabas G, Wente MN, et al. Incidence, risk factors, and prevention of biliary tract injuries during laparoscopic cholecystectomy in Switzerland. World J Surg 2001;25(10):1325-1330. DOI: 10.1007/s00268-001-0118-0.

5. Henneman D, da Costa DW, Vrouenraets BC, et al. Laparoscopic partial cholecystectomy for the difficult gallbladder: a systematic review. Surg Endosc 2013;27(2):351-358. DOI: 10.1007/s00464-012-2458-2.

6. Michalowski K, Bornman PC, Krige JE, et al. Laparoscopic subtotal cholecystectomy in patients with complicated acute cholecystitis or fibrosis. Br J Surg 1998;85(7):904-906. DOI: 10.1046/j.13652168.1998.00749.x.

7. Sinha I, Smith ML, Safranek P, et al. Laparoscopic subtotal cholecystectomy without cystic duct ligation. Br J Surg 2007;94(12):1527-1529. DOI: 10.1002/bjs.5889.

8. Nakajima J, Sasaki A, Obuchi T, et al. Laparoscopic subtotal cholecystectomy for severe cholecystitis. Surg Today 2009;39(10): 870-875. DOI: 10.1007/s00595-008-3975-4.

9. Harilingam MR, Shrestha AK, Basu S. Laparoscopic modified subtotal cholecystectomy for difficult gall bladders: A single-centre experience. J Minim Access Surg 2016;12(4):325-329. DOI: 10.4103/0972-9941.181323.

10. Palanivelu C, Rajan PS, Jani K, et al. Laparoscopic cholecystectomy in cirrhotic patients: the role of subtotal cholecystectomy and its variants. J Am Coll Surg 2006;203(2):145-151. DOI: 10.1016/ j.jamcollsurg.2006.04.019.

11. Horiuchi A, Watanabe Y, Doi T, et al. Delayed laparoscopic subtotal cholecystectomy in acute cholecystitis with severe fibrotic adhesions. Surg Endosc 2008;22(12):2720-2723. DOI: 10.1007/s00464-0089879-y.

12. Singhal T, Balakrishnan S, Hussain A, et al. Laparoscopic subtotal cholecystectomy: initial experience with laparoscopic management of difficult cholecystitis. Surgeon 2009;7(5):263-268. DOI: 10.1016/ S1479-666X(09)80002-4.

13. Tamura A, Otsuka Y, Tsuchiya $M$, et al. Perioperative and long follow up evaluation of laparoscopic subtotal cholecystectomy. HPB 2012;2:338.

14. Ali L, Bashir T, Bashir S. Laparoscopic subtotal cholecystectomy in difficult cholecystitis - our experience. Pak J Med Health Sci 2015;9(3):917-919.

15. Peker KD, Alis H. Laparoscopic subtotal cholecystectomy could be an alternative to conversion. Med J Bakirkoy 2017;13(3):113-117. DOI: 10.5350/BTDMJB201713301.

16. Elshaer M, Gravante G, Thomas K, et al. Subtotal cholecystectomy for "difficult gallbladders": systematic review and meta-analysis. JAMA Surg 2015;150(2):159-168. DOI: 10.1001/jamasurg.2014.1219.

17. van Dijk AH, Donkervoort SC, Lameris W, et al. Short- and longterm outcomes after a reconstituting and fenestrating subtotal cholecystectomy. J Am Coll Surg 2017;225(3):371-379. DOI: 10.1016/ j.jamcollsurg.2017.05.016.

18. Kim Y, Wima K, Jung AD, et al. Laparoscopic subtotal cholecystectomy compared to total cholecystectomy: a matched national analysis. J Surg Res 2017;218:316-321. DOI: 10.1016/j.jss.2017.06.047.

19. Chowbey P, Sharma A, Goswami A, et al. Residual gallbladder stones after cholecystectomy: a literature review. J Minim Access Surg 2015;11(4):223-230. DOI: 10.4103/0972-9941.158156.

20. Jeong IO, Kim JY, Choe YM, et al. Efficacy and feasibility of laparoscopic subtotal cholecystectomy for acute cholecystitis. Korean J Hepatobiliary Pancreat Surg 2011;15(4):225-230. DOI: 10.14701/ kjhbps.2011.15.4.225.

21. Shingu $Y$, Sakamoto E, Norimizu S. Preoperative prediction of laparoscopic subtotal cholecystectomy for severe cholecystitis. Am J Gastroenterol 2015;1:S27.DOI: 10.14309/00000434-201510001-00064. 